\title{
A GENERAL ALGORITHM FOR LIMIT SOLUTIONS OF PLANE STRESS PROBLEMS
}

\author{
HoON HUH' and WeI H. YANG \\ Department of Mechanical Engineering and Applied Mechanics, \\ The University of Michigan. Ann Arbor, MI 48109, U.S.A.
}

(Receited 8 August 1990; in retised form 14 December 1990)

\begin{abstract}
A computational approach to limit solutions is considered most challenging for two major reasons. A limit solution is likely to be non-smooth such that certain non-differentiable functions are perfectly admissible and make physical and mathematical sense. Moreover. the possibility of non-unique solutions makes it difficult to analyze the convergence of an iterative algorithm or even to define a criterion of convergence. In this paper, we use two mathematical tools to resolve these difficulties. A duality theorem defines convergence from above and from below the exact solution. A combined smoothing and successive approximation applied to the upper bound formulation perturbs the original problem into a smooth one by a small parameter $\varepsilon$. As $\varepsilon \rightarrow 0$, the solution of the original problem is recovered. This general computational algorithm is robust such that from any initial trial solution, the first iteration falls into a convex hull that contains the exact solution(s) of the problem. Unlike an incremental method that invariably renders the limit problem ill-conditioned, the algorithm is numerically stable. Limit analysis itself is a highly efficient concept which bypasses the tedium of the intermediate elastic-plastic deformation and seeks the most important information directly. With the said algorithm, we have produced many limit solutions of plane stress problems. Certain non-smooth characters of the limit solutions are shown in the examples presented. Two well-known as well as one parametric family of yicld functions are used to allow comparison with some classical solutions.
\end{abstract}

\section{INTRODUCTION}

The theory of plasticity has become an important scientific foundation for optimal design of metal structures and machinery. It is not that modern designs are intended to function beyond the elastic limit, they are not. Plastic designs are more uniform in strength and therefore less prone to weak links. They are more accurate in predicting failure conditions and thus provide precise safety factors for overloading caused by natural disasters and human errors. Plastic designs also lead to savings of materials and hence a reduction in weight and cost. All these advantages should have attracted a crowd of engineers to research and practice the theory of plasticity. But like good things in life, it carries a higher price tag. Plastic analysis and designs are much more difficult than their elastic counterpart. Plastic constitutive relations are inherently non-linear and non-one-to-one. Mathematical difficulty and the need for large-scale computation involved in solving meaningful problems have encumbered the early development of plasticity and still make the progress of this branch of mechanical science slow.

Recent explosive advances in the capacity and speed of computers have made plastic analysis computationally practical. Growing demands for crashworthy vehicles, accidenttolerant nuclear facilities and earthquake-resistant structures have made plastic designs indispensable. Renewed interest in plasticity has been abreast. Two types of analyses, incremental and asymptotic, have been promoted and pursued.

The asymptotic approach to plasticity is known as the limit analysis. Although the theory was first developed in the 1950s in an ad hoc manner, recent work on limit analysis (Maier et al., 1972; Martin, 1975; Strang et al., 1978; Temam, 1984; Yang, 1987) has merged it with an exciting field in modern mathematics known as mathematical programming (Luenberger, 1984). Benefitting from cross-fertilization and progress made in other fields, a concise architecture of limit analysis has now emerged with new physical interpretation, rigorous mathematical formulation and efficient computational methodology. We shall briefly describe its framework as a mechanics problem, interpret a model 
of asymptotic behavior of a material, state a variational principle of duality. develop a computational algorithm and finally present some examples. Although the concepts apply to general limit analysis, all discussions in this paper pertain only to the class of plane stress problems.

\section{LIMIT ANALYSIS OF PLANE STRESS PROBLEMS}

From a vector (or function) space point of view, a solution of a mechanics problem lies in the intersection of three fundamental sets, namely the statically-admissible set $S$, the constitutively-admissible set $C$ and the kinematically-admissible set $K$. If the intersection is empty. there exists no solution. If it consists of a single point. the solution is unique. Otherwise, there is a set of feasible solutions of which one maly be the most preferred (optimal). The criterion for choosing the optimal solution is facilitated by an objective function. Since $S \cap C \cap K$ is a subset of $S \cap C$, the optimal solution contained in the former is obviously in the latter. The primal (or natural) formulation of a limit analysis problem seeks an extreme point in $S \cap C$ as its optimal solution.

The asymptotic behavior of some metals is exhibited by their great ductility. A onedimensional model uses a hardening function. such as that of Ramberg and Osgood (1943), to describe the results of a tensile experiment. from the initial yield point to the subsequent (higher or equal) yield points. Classical limit analysis assumes a perfectly-plastic material such that it does not harden or the hardening function is a constant. the yield stress. We shall remove this assumption and only require that the hardening curve asymptotically approaches a constant state of stress, the true limit of the material's stress-bearing capacity. A three-dimensional model generalizes the concept of a yield point to a surface in the space of the stress matrices, $R^{1 \times}$. The surfices representing the initial and subseyuent yielding behavior are described by a yield function with parancters to account for the hardening behavior. The assumption that the hardening function hats a constant asymptote implies the existence of an envelope that encloses all yield surfaces. We shall call this envelope the asymptotic yield surface. The states of stresses bounded by the envelope are feasible and a stress state outside the evelope cannot be attained. This model of plastic behavior is called asymptotically perfect.

The elastic property of the material is not needed in the analysis but nor is it explicitly excluded by the constitutive inequality. We only assume a large elastic modulus so that the deformation remains small before an impending failure. This departure from the rigid, perfectly-plastic model of classical limit analysis does not change the nature of the problem. only broadens its applicability. A Lagrangian coordinate system is used to describe deformation and equilibrium about the undeformed state.

A state of plane stress in a thin sheet is represented by a symmetric $2 \times 2$ matrix function,

$$
\sigma=\left[\begin{array}{ll}
\sigma_{x x} & \sigma_{x y} \\
\sigma_{y x} & \sigma_{y y}
\end{array}\right], \quad \sigma_{y,}=\sigma_{x y}
$$

whose components are real functions of $(x, y)$ and its eigenvalues are denoted by $\sigma_{1}$ and $\sigma_{2}$, also functions of $(x, y)$. A stress distribution in the sheet. being a matrix function defined in a domain $D$ in the $(x, y)$-plane, is regarded as a point in the function space $R^{2 \times 2}(D)$. The asymptotic behavior of the sheet for the limit analysis to be presented in this paper is modeled by

$$
\|\sigma\|_{(\beta)}=\sqrt{\sigma_{i}^{2}-\beta \sigma_{1} \sigma_{2}+\sigma_{2}^{2}} \leqslant \sigma_{0}, \quad-2<\beta<2
$$

where $\sigma_{0}$ is a constant, the asymptotic yield stress. and the range of the parameter $\beta$ guarantees convexity of the yield functions defined by the $\beta$-norm. The use of a norm notation appropriately reflects the intended convexity and conveys the meaning of a bound on the stress matrix. The $\beta$-norm reduces to the well-known von Mises yield function when 


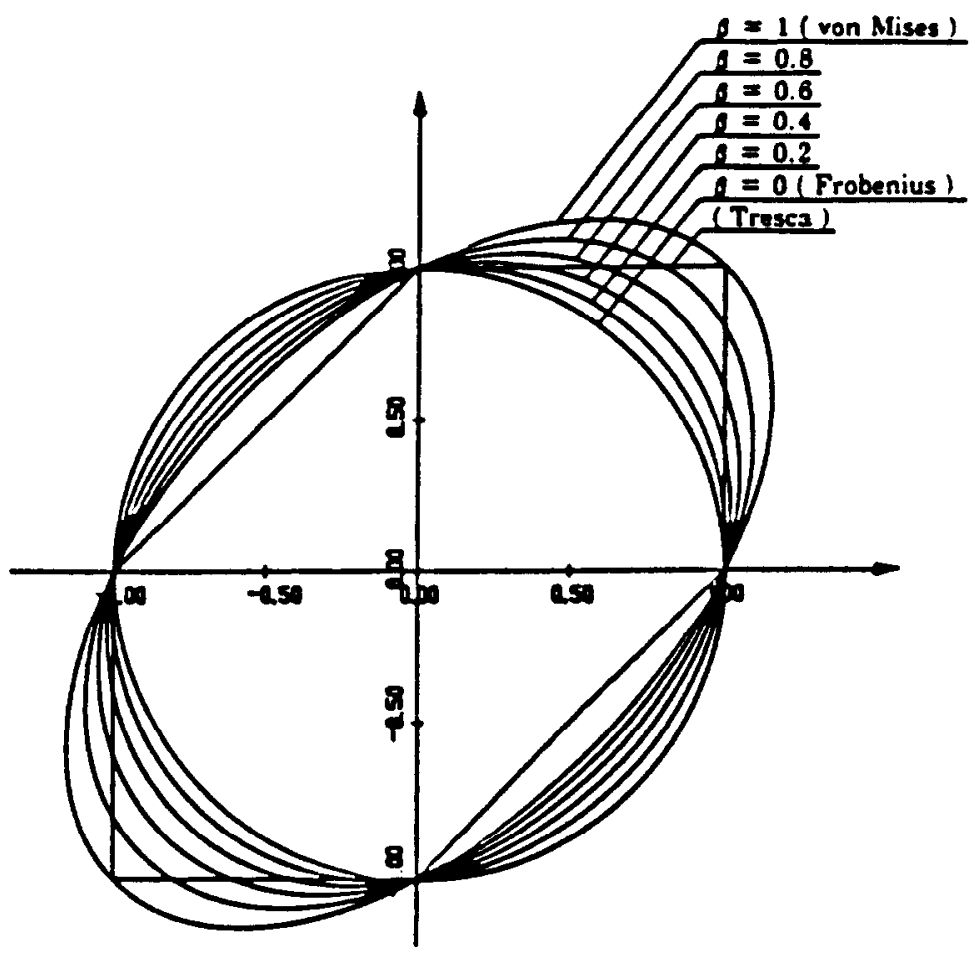

Fig. 1. Family of $\beta$-norm functions and the Tresca yield function.

$\beta$ is equal to unity. The case $\beta=0$ corresponds to the Frobenius norm of a matrix and is sometimes chosen for its mathematical convenience. The proper choice of $\beta$ is of course the best fit to the experimental data of a specific material. The $\beta$-norm family of yield criteria for the range $0 \leqslant \beta \leqslant 1$ and the well-known Tresca criterion are shown in Fig. I. Inequality (2) defines the constitutively-admissible set $C \subset R^{2 \times 2}(D)$.

The statically-admissible solutions of a plane stress problem satisfy the equilibrium equation $\nabla \cdot \sigma=0$ in the domain $D$ and the static boundary condition $\sigma \cdot \mathbf{n}=\mathbf{t}$ on the part of the boundary $i D$, where a given traction vector $t$ is prescribed. These solutions form the statically-admissible set $S \subset R^{2 \times 2}(D)$. A limit analysis problem secks an extreme point in $S \cap C$ that maximizes the applied load in its proportional form, $q t$, where $y$ is a positive, real scaling factor. The constrained maximization of the objective functional $q(\sigma)$ in the form

$$
\begin{array}{ll}
\text { maximize } & q(\sigma) \\
\text { subject to } & \nabla \cdot \sigma=0 \text { in } D \\
& \sigma \cdot \mathbf{n}=q \mathbf{t} \text { on } i D_{s} \\
& \|\sigma\|_{(\beta)} \leqslant \sigma_{0}
\end{array}
$$

defines the primal formulation of limit analysis for the plane stress problems. Since the equilibrium equation is linear and the constitutive inequality is convex, the intersection $L=S \cap C$ is convex. Problem (3) is a convex programming in the function space $R^{2 \times 2}(D)$. It is also called the lower-bound formulation in plasticity and $L$ is called the lower-bound solution set since every point in $L$ corresponds to a value of $q$ either lower or equal to the maximum value $q^{*}$ sought.

\section{DUALITY}

A convex programming problem has a dual problem whose minimum is equal to $q^{*}$. To construct the dual problem of (3), we begin with the weak equilibrium equation 


$$
\int_{0} \mathbf{u} \cdot(\nabla \cdot \sigma) \mathrm{d} A=0
$$

where $\mathbf{u}$ is an arbitrary function in $R^{2}(D)$ with the physical interpretation of an admissible velocity function. An admissible u which satisfies the kinematic conditions (homogeneous for the problems in this paper) on the part of the boundary $D_{k}$ complementary to $D_{s}$ and derives meaningful quantities under a generalized divergence theorem will lead to the equivalent variational statement

$$
\int_{D} \sigma: \varepsilon \mathrm{d} A=q \int_{i D_{r}} \mathbf{t} \cdot \mathbf{u} \mathrm{d} S
$$

where $\varepsilon=\frac{1}{2}\left(\nabla \mathbf{u}+\nabla \mathbf{u}^{\prime}\right)$ is the $2 \times 2$ strain rate matrix and : denotes the inner product operator between two matrices. All such functions, u, form the kinematically-admissible set $K \subset R^{2}(D)$. Since certain non-differentiable functions are admissible in (5), this relaxed variational principle greatly enlarged the set $K$ from the set of compatible strains defined in the theory of elasticity (Timoshenko and Goodier, 1970). Since u appears homogeneously in (5). implicitly in $\varepsilon$ on the left-hand side. we may normalize the boundary integral such that

$$
\int_{i n,} \mathbf{t} \cdot \mathbf{u d} S=1
$$

which also implies that the integral does not vanish and $\sigma: \varepsilon>0$. We shall add (6) to the conditions of kinematic admissibility which will be defined more preciscly.

A generalized Hölder inequality

$$
\left.|\sigma: \varepsilon| \leqslant\|\sigma\|_{(\beta)}\|\varepsilon\|_{1}, \beta\right)
$$

was recently established by Yang (1991), where the $(-\beta)$-norm is called the dual of the $(\beta)$-norm. In terms of the eigenvalues $\varepsilon_{1}$ and $\varepsilon_{2}$ of the $2 \times 2$ strain rate matrix $\varepsilon$,

$$
\|\varepsilon\|_{1-p)}=\frac{1}{\sqrt{1-(\beta / 2)^{2}}} \sqrt{\varepsilon_{1}^{2}+\beta \varepsilon_{1} \varepsilon_{2}+\varepsilon_{2}^{2}}
$$

Inequality (7) is sharp, meaning that equality holds when $\varepsilon$ is chosen to be proportional to the gradient of the yield function. This sharpness condition

$$
\varepsilon=k \nabla\|\sigma\|_{(\beta)}
$$

is the well-known normality condition of Drucker (1959) in plasticity, where $k$ is a proportional factor. Represented in the principal stress space of Fig. 1, is a principal strain rate in the form of a vector $\varepsilon=\left(\delta_{1} \varepsilon_{2}\right)$ associated with a state of yield stress $\left(\sigma_{1} \sigma_{2}\right)$ (a point on the yield surface), which is normal to the yield surface.

Using (2), (5), (6) and (7), we can establish a sharp upper bound to the functional $q(\sigma)$ by the sequence of inequalitics

$$
q=\int_{D} \sigma: \varepsilon \mathrm{d} A \leqslant \int_{D}\|\sigma\|_{(m)}\|\varepsilon\|_{(-\infty)} \mathrm{d} A \leqslant \sigma_{0} \int_{D}\|\varepsilon\|_{(-D)} \mathrm{d} A=\bar{q}(\mathrm{u}),
$$

where $\bar{q}(\mathbf{u})$, the upper bound functional, depends only on the kinematic functions $\mathbf{u} \in K$. The correct choice of $K$ is studied in the deeper and still ongoing research of functional analysis and calculus of variations (Cesari et al.. 1988). A proof of the existence of an absolute minimum for functionals like $\bar{q}(u)$ has been obtained. 
Based on the sharp inequalities in (10) and the existence of the absolute minimum of $\bar{q}(u)$. we may state the dual problem (or the upper bound formulation)

$$
\begin{array}{ll}
\text { minimize } & \bar{q}(\mathrm{u}) \\
\text { subject to } & \bar{q}(\mathrm{u})=\sigma_{0} \int_{D}\|\varepsilon\|_{(-\beta)} \mathrm{d} A, \\
& \int_{i D_{*}} \mathrm{t} \cdot \mathrm{u} \mathrm{d} S=1, \\
& \text { kinematic boundary conditions on } 2 D_{k} .
\end{array}
$$

The smallest function space, whose elements satisfy the constraints in (11) and at least one of them produces the absolute minimum of the objective functional, defines $K$. When the absolute minimum of $\bar{q}(u)$ is attained, we can realize the duality relation

$$
\max _{\sigma \in L} q(\sigma)=q^{*}=\min _{\mathbf{u} \in \mathcal{K}} \bar{q}(\mathbf{u})
$$

In reality, only in the simplest cases can (11) be solved exactly. General solutions of (11) must be obtained numerically. In this paper, the upper bound functional is first discretized, then a combined smoothing and successive approximation algorithm [see Ben-Tal et al. (1991)] is used to solve the finite-dimensional minimization problem. We have successfully obtained many plane stress limit solutions, some of which are presented in a later section.

\section{FINITE-DIMENSIONAL APPROXIMATION}

We shall use a finite element method [see Zienkiewicz (1977)] to discretize the dual problem (11) and to reduce it to a convex programming problem in a finite-dimensional space $R^{n}$, where $n$ is the total number of discrete variables. The standard three-node triangular elements are chosen to discretize the domain. The velocity field in each element is approximated by a linear function. From this assumed elemental velocity function the strain rate is therefore a constant matrix in each element. The integral representing the upper-bound functional $\bar{q}(u)$ in (11) is approximated by a sum

$$
\bar{q}(U)=\sum_{e=1}^{E} \sqrt{U^{2} A_{e} U}
$$

where $U$ is the vector representation of the velocity function $\mathbf{u}_{,} t$ transposes a vector, $A_{f}$ is the elemental "stiffness matrix" generated by the finite element method and the integer $E$ is the total number of elements. From the elemental view point, the velocity vector and the matrix $A_{e}$ have dimensions 6 and $6 \times 6$, respectively. However, the scalar product $U^{\prime} A_{e} U$ in each term of the sum is interpreted as a product formed in $R^{n}$ where $U \in R^{n}$ is the global velocity vector and $A_{e}$ is embedded in an $n \times n$ null matrix.

Similarly, the normalization equation $\int t \cdot u d S=1$ is approximated by $C^{\prime} U=1$, where $C \in R^{n}$ is a constant vector. The finite-dimensional approximation of (1I) takes the form

$$
\begin{array}{ll}
\text { minimize } & \dot{q}(U) \\
\text { subject to } & \tilde{q}(U)=\sum_{c=1}^{\varepsilon} \sqrt{U^{t} A_{e} U} \\
& C^{\prime} U=1
\end{array}
$$

where the parameters $\sigma_{0}$ and $\beta$ as well as the kinematic boundary conditions are absorbed into the matrices $A_{e}$ and the vector $C$. 
It can easily be shown that each $A_{e}$ is positive semi-definite and $\sqrt{U^{\mathrm{t}} A_{e} U}$ is a convex function in $R^{n}$. Since the sum of convex functions is convex. $q(C)$ is convex and has a unique minimum value.

One last obstacle is still in the path of a numerical solution of (14). The matrices $A_{e}$ are only positive semi-definite such that the product $C^{-t} A_{c} \cdot C$ may vanish for some non-trivial vectors $U$. The square-root functions are not differentiable at the zero value of their argument. This non-smoothness in the derivatives may cause trouble in the gradient or Newton-like algorithms of minimization, e.g. Broydon (1967). An attempt to evaluate the gradient of a square root near a zero argument may cause computational overflow. We chose a small real number $\varepsilon$ as the smoothing parameter to remove this difficulty. It led to a perturbed objective function

$$
\bar{q}(U, \varepsilon)=\sum_{i=1}^{E} \sqrt{C^{t} A_{\varepsilon} U+\varepsilon^{2}}
$$

which is differentiable everywhere for $\varepsilon \neq 0$ and remains convex. The perturbed function recovers its original value as $\varepsilon \rightarrow 0$.

Using a Lagrangian multiplier $\lambda$ to convert the constrained problem (14) into an unconstrained one with the perturbed objective function in (15), we minimize instead

$$
\phi\left(C^{\prime}\right)=\tilde{q}\left(C^{\prime}, \varepsilon\right)-i\left(C^{\prime} C^{\prime}-1\right)
$$

whose minimum solution satisfies the condition $i \phi^{\prime} r U_{i}=0, i=1.2 \ldots \ldots$. After performing these partial derivatives, a system of equations is obtained. In matrix notation, the system

$$
A l=i C
$$

has a global stiffness matrix

$$
A=\sum_{i=1}^{t} \frac{d}{U^{i} d U+\theta^{2}}
$$

Which is regarded as a constant matrix in each iteration and is updated from iteration to iteration. For a given vector $U$. a constant matrix $A$ caln be evaluatted. Equation (17) is treated in each iteration as a linear system to be solved repeatedly with an inner and an outer iterative sequence.

Symbolically, a solution maty be expressed in terms of the inverse of $A$ (which is not computed in practice) such that

$$
U_{n:}=i A^{-1} C
$$

where $\lambda$, still an unknown quantity, can be evaluated by the condition $C^{\prime} \dot{U}=1$ to obtain

$$
i=\frac{1}{C^{-1} A^{-1}}
$$

The outer iteration is associated with a decreasing sequence of $\varepsilon$. With each fixed value of $\varepsilon$, an inner iteration begins with a known vector $U$ so that the stiffness matrix $A$ in (17) can be evaluated initially. The solution of (17) in each inner iteration is used in a feedback loop to update $A$ and $i$. A converged $U_{t}$ under a suitable stopping criterion terminates an inner iteration loop. Then $\varepsilon$ is reduced and another inner iteration begins. Only in the first inner iteration is an initial vector $U^{(0)}$ assumed. The subsequent inner iterations use the converged solution for the previous $\varepsilon$ as its initial vector. We need only a few values of $\varepsilon$ to extrapolate to the limit. $\varepsilon \rightarrow 0$. From any initial vector $U^{(n)}$. the subsequent iterates are 
locked in a certain convex hull defined by the data of the discrete problem (14). This robust initial convergence and the rate of convergence for the subsequent iterations are discussed in Ben-Tal et al. (1991).

\section{CIRCULAR SHEET WITH A CONCENTRIC HOLE}

The first application of a newly-developed methodology must be a test of its ability to reproduce a known result. A circular sheet with a concentric hole under axisymmetric loading has an exact solution obtained by a non-numerical method for $\beta=1$, the von Mises case. The exact solution can serve as a comparison to test our numerical algorithm.

We choose the exact limit solution of Kachanov (1971) as the bench mark for comparison. A circular sheet of radius $b$ with a centered hole of radius $a$ is subjected to an axisymmetric tensile loading at the outer radius. The simplicity of this problem also allows both primal and dual solutions to be obtained computationally. The numerical solutions from maximization and minimization offer a self-check for accuracy and duality. They are shown in Table 1 for various $a / b$ ratios and a fixed $\beta=1$. The small duality gaps between the upper and lower bound solutions are due to numerical errors inherited from a terminating criterion.

The exact solution of Kachanov (1971), shown in Fig. 2 for the von Mises yield function, falls within the gaps, a strong verification of the dual variational principle and the algorithm. The exact solution for the Trescal criterion. plotted as the dotted curve below the solid curve for the von Mises criterion, confirms a long standing conjecture that a larger constitutively-admissible set will not result in a lower collapse load. Both curves approach the value $q^{*}=1$ continuously as $a / h \rightarrow 0$, suggesting that a small imperfection in the material does not effect its load-carrying capacity. This comforting property of ductile material differs markedly from the britule fracture theory derived from small imperfections.

The velocity fields under the collapse conditions as functions of radial positions are plotted in Fig. 3 for valrious $a / h$ ratios. Except for the calse $a / b=0$ (no hole), strain rattes in the r-direction (du/dr) are negative nealr the hole. This is duc to the uniaxial nature of the stress field in the circumferential direction near the hole, producing thinning in both the $r$ - and z-directions.

If the loading is applied at the inner radius $a$, the domain $a \leqslant r \leqslant b$ can be regarded as the flange portion of a circular sheet in a deep drawing process in which the sheet is drawn into a cup by a die and punch press [see Avitzur (1968)]. The key question in this manufacturing process is the ability of the material to be drawn for a given flange size. The process will be suceessful only if the entire hange can undergo plastic deformation due to the inward drawing force. A failure of the process is called choking in that only the innermost part of the flange deforms plastically. The rest of the flange remains stationary, resulting in rapid thinning and thus tearing near $r=a$. The largest flange (minimum $a / b$ ratio) that can be drawn increases with increasing $\beta$ for a material modeled by the $\beta$-norm yield criteria, as shown by the dotted choking limit curve at the top of all curves in Fig. 4 . We extended

Table I. Upper and lower bound solutions for a circular sheet of radius $b$ with a concentric hole of radius $a$

\begin{tabular}{lccc}
\hline$a h$ Upper bound $(j)$ & Lower bound $(q)$ & Gap $(\%)$ \\
\hline 0.0 & 1.0 & 1.0 & 0.0 \\
0.05 & 0.99306348 & 0.99242223 & 0.064 \\
0.1 & 0.97192178 & 0.97062629 & 0.133 \\
0.2 & 0.89543868 & 0.89421282 & 0.137 \\
0.3 & 0.79202212 & 0.79113852 & 0.112 \\
0.4 & $0.676+4796$ & 0.67585636 & 0.087 \\
0.5 & 0.55720578 & 0.55660137 & 0.108 \\
0.6 & 0.43793322 & 0.43776690 & 0.038 \\
0.7 & 0.32176233 & 0.32168261 & 0.025 \\
0.8 & 0.20959000 & 0.20953326 & 0.027 \\
0.9 & 0.10235899 & 0.10228872 & 0.069 \\
1.0 & 0.0 & 0.0 & 0.0 \\
\hline
\end{tabular}




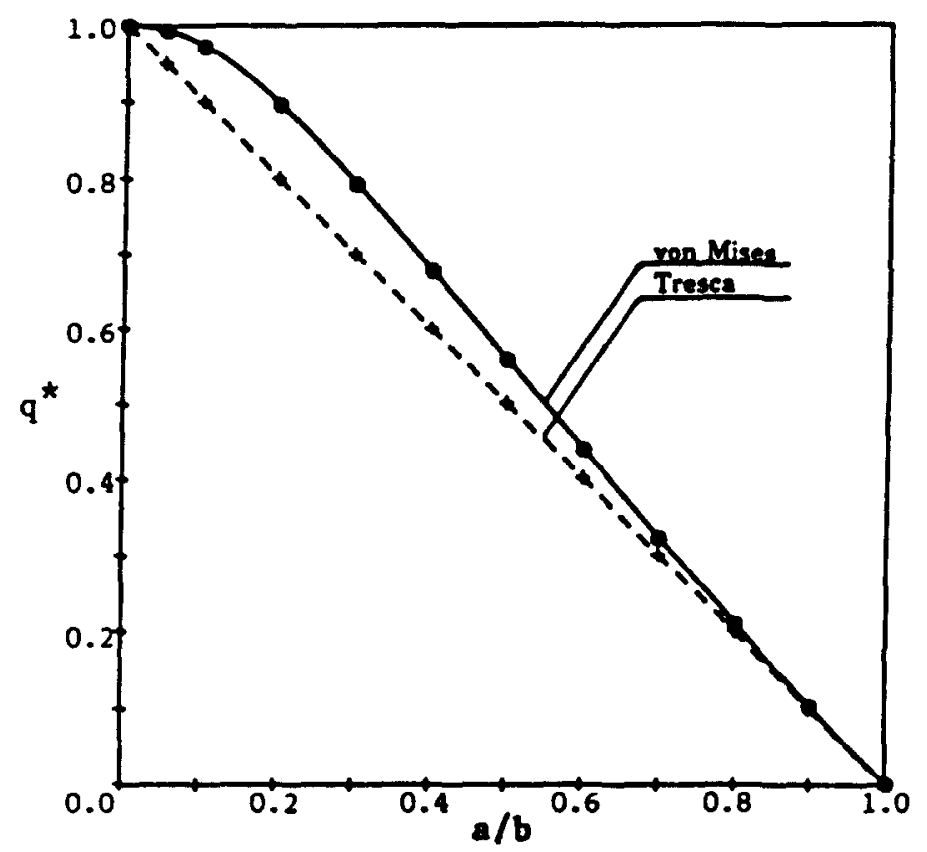

Fig. 2. Collapse loids of circular sheet with concentric hole of radius a under axisymmetric loading at its outer radius $b$.

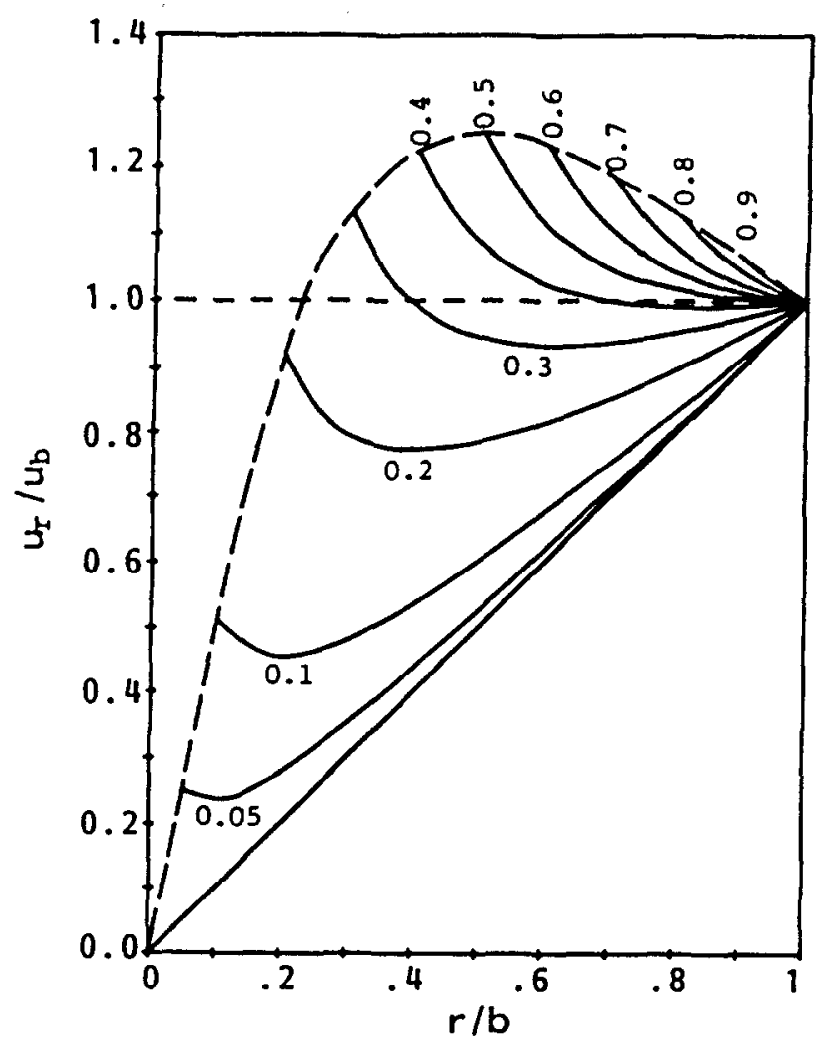

Fig. 3. Velocily distributions in circular sheets with various hole sizes. 


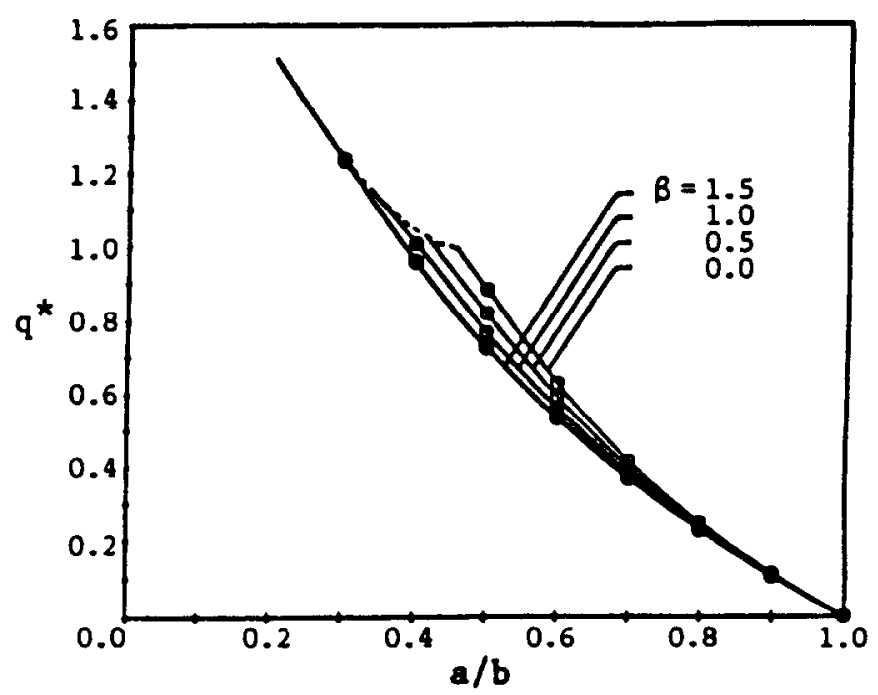

Fig. 4. Drawing loads and hange limits of cup drawing process.

the computation to the case $\beta=1.5$. The $\beta$-family resembles Hill's (1950) anisotropic models of rolled sheet metals with a parameter $R$. The trend of formability in the Hill and i models of materials are similar.

\section{A STRIP WITII AN FLLIPTIC FIGLE OR TWO SIMIELLIPTIC NOTCHES}

Elastic solutions of a tension strip with a centered elliptic hole or two oppositelylocalted semielliptic notches hatve been studied in great detail as regards the elfect of stress concentration in elasticity. As the ratio of the principal axes of the ellipse $h / a$ approaches zero, the singular stress ficld near a crack tip is of great signilicance in predicting britte fracture [see Williams (1957)]. Such an approach is invalid for ductile materiats like metals. In plasticity, the stresses are bounded and therefore no severe concentration may occur, let alone a singular stress ficld. Instead, strains may concentrate. The forms of concentration in plastic deformation difter greatly from that of a singular stress field in elasticity. A kink in a beam. a neck in a tensile bar and a slip band in a body have mathematical representations of non-differentiable or even discontinuous functions. To capture these phenomena computationally is challenging.

Let the transverse axis $a$ of the ellipse be made equal to one-fourth of the width of the thin strip. Several ratios of the principal axes, $b / a$, of the hole or notches between 0 and 2 are chosen for our computation of collapse loads and velocity fields. The collapse loads are shown in Fig. 5, from which two phenomena are observed. First, the strip with a hole is always weaker than that with two notches of the same cut-out area. This means that an interior flaw in a material is more serious than a comparable boundary defect. When the ratio b/a decreases for a hole or notch, a lesser amount of material is being cut out and the collapse load increases. The second observation agrees with another long-standing conjecture in plasticity, that added weightless material to a structure will not decrease its strength. Adding material to make a hole or a notch into a crack is of course the most inefficient use of material. Nevertheless, the results fulfill the prophecy of the conjecture. When the linear theory of elasticity is used, one shall reach just the opposite conclusion that a strip with a crack is infinitely weaker than a strip with a hole of the same width. Ductile failure of a structure cannot be predicted by the linear theory of elasticity.

The collapse modes in terms of velocity fields and grid distortions are shown in Figs 6 and 7 for an internal crack and two elliptic notches, respectively. By symmetry, only a quadrant of the domain is presented. The velocity fields show that the major portion of the sheet undergoes rigid body motion. Deformation is concentrated in narrow bands near the 


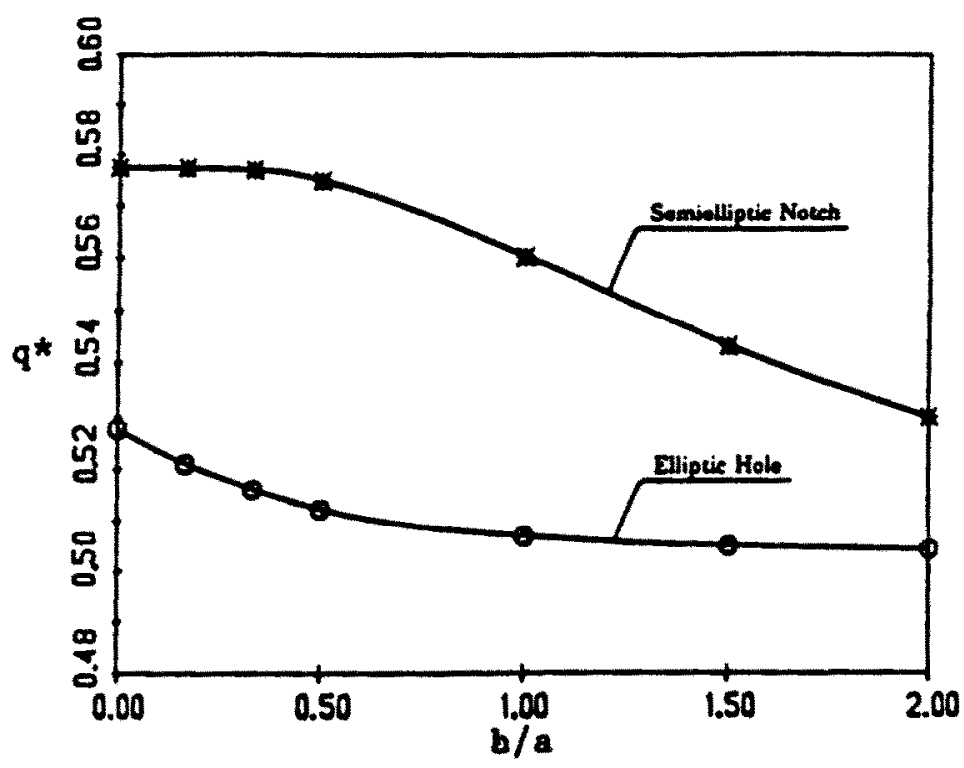

Fig. 5. Limit loads of tension strips with elliptic hole or notches.
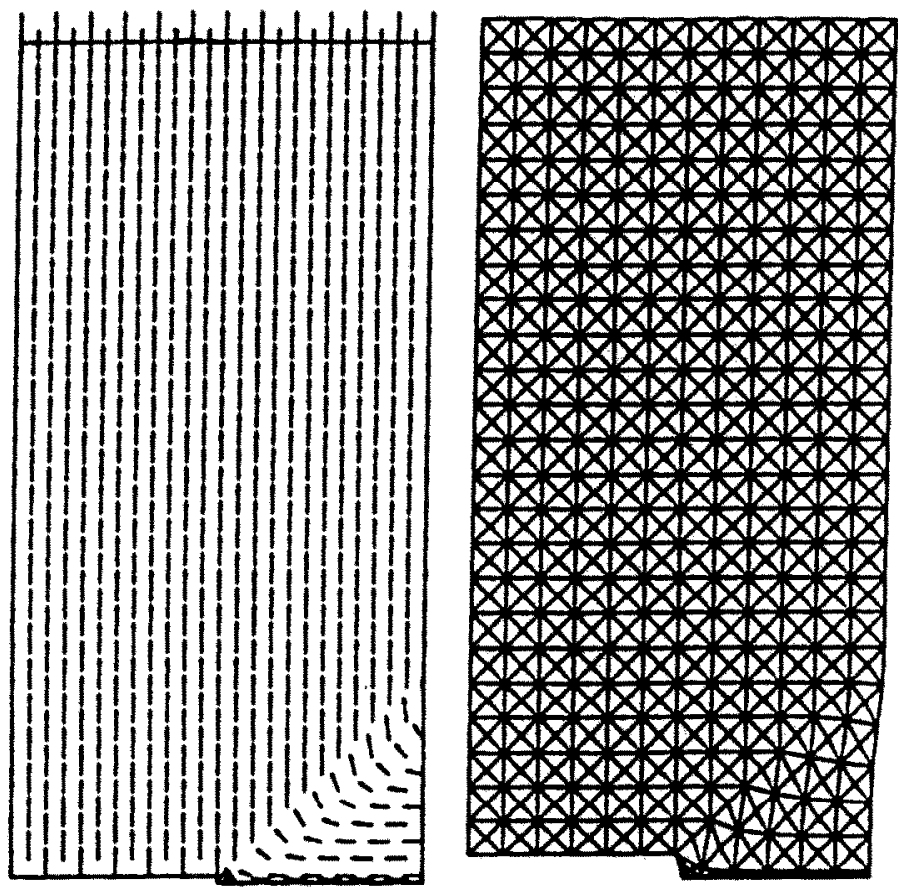

Fig. 6. Velocity field and deformed grid as representations of collapse modes of a cracked tension strip.

weakest cross-section. This common phenomenon in plastic deformation is often referred to as localization by Rice (1976). The non-smooth functions involved in the solutions can cause trouble in some computational methods. Our algorithm has successfully captured this non-smoothness in the limit solutions. The results confirmed certain classical solutions and extended the range of parameters to cover new cases. 

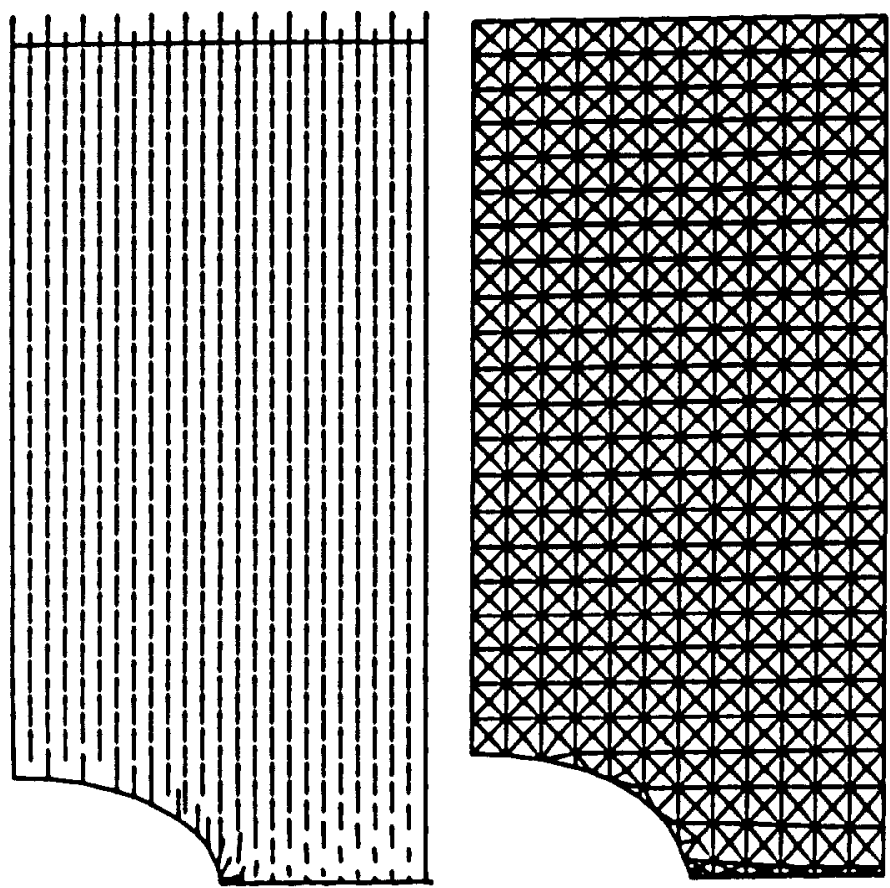

Fig. 7. Velocity field and deformed grid as representations of collipse modes of a notched tension strip.

\section{CONCLUSION}

A general algorithm has been developed and successfully tested for limit analysis of plane stress problems. The algorithm is built on sound physical, mathematical and computational foundations. The parametric $\beta$-norm yield functions can certainly fit the behavior of a wide class of materials. The duality theorem helps to discern the direction and rate of convergence when an iterative approach is applied to either the primal or the dual formulation. When the primal and dual problems are solved simultaneously, the closing of the duality gatp provides the true indicator of convergence, especially in the case when the iterative solution vector wanders indefinitely between equally-acceptable but non-unique solutions. The combined smoothing and successive approximation method homes in robustly on a correct non-smooth optimal solution.

The example problems presented are certainly non-trivial. Even the seemingly-trivial axisymmetric problem provides the comparison with the exact solutions needed for checking the algorithm. It reveals the effect of the hole size. As the radius of the hole approaches zero, the strength of the sheet uniformly approaches that of a flawless sheet. Insensitivity to small defects is a very desirable property of the ductile materials. It also produced new results for a wide class of $\beta$-family materials to indicate the trend of formability in the deep drawing process. Furthermore, the collapse loads for the von Mises criterion being equal or greater than that for the inscribed Tresca criterion lends credence to a long-standing conjecture that a larger constitutively-admissible set will not result in a smaller collapse load.

The problem of the holed or notched tension strip reveals again a major difference between elastic and plastic response towards material defects. Geometric variations of the hole and notch with the limiting cases of cracks confirm another well-known conjecture in plasticity, that added weightless material will not weaken the structure. The non-smoothness of the solutions captured by our computation further confirmed the good mathematical analysis that goes into the algorithm.

The large mesh systems. multitudes of material and geometric parameter variations and double-sequenced iterative loops should make the computational task executed for this 
paper comparable to any major project. The general algorithm for limit analysis has performed well for the plane stress problems with very acceptable accuracy at a quite modest cost. Computations for each example problem and their parametric variations converged under 30 total iterations with $\| \Delta l \psi,<0.001$ between two consecutive iterative solutions as the stopping criterion. The outer sequence uses only three values of $\varepsilon, 0.1$. 0.01. 0.001. The accumulative CPU time on an Amdahl 5860 computer for all example problems was a mere $10 \mathrm{~min}$. We are now considering implementing the algorithm on a microcomputer.

\section{REFERENCES}

Avitzur. B. (1968), Metal Forming: Processs and Andisis. McGraw-Hill. Scarborough. CA.

Ben-Tal. A., Teboulle. M. and Yang. W. H. (1991). A combined smoothing and sucessive approximation method for limit analysis. To appear in J. Appl. Math. Optimis.

Broydon. C. G. (1967). Quasi-Newton methods and their applications to function minimization. Math. Comp. 21. $368-381$.

Cesari. L.. Brandi. P. and Salvadori. A. (1988). Existence theorems for multiple integrals of calculus of variations for discontinuous solutions. Anm. M/at. Pura Appl, $152(+1,95121$.

Drucker. D. C. (1959). A detinition of stable inelastic material in the mechanics of eontinual. J. Appl. W/ech. 81, $101-106$.

Hill. R. (1950). The Mathematical Theors of Plasticits. Clarendon Press, Oxford.

Kachanov. L. M. (1971). Fondetiens of whe Theory of Plasticit!. North-Hoiland. Amsterdatm.

Luenberger. D. G. (1984). Limear and Vomlinear Programminu. 2nd edn. Addison Wesley. Reidding. MA

Maier, G., Zavelanti. A. and Beneditti. D. (1972). A linite element approatch to optimal design of plastic structures in plane stress. Int. $J$. Numer. Meth. Enumy $4,455+73$.

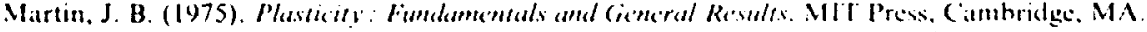

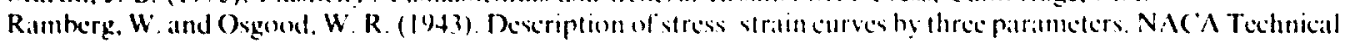
Note Nu. $\%(02$.

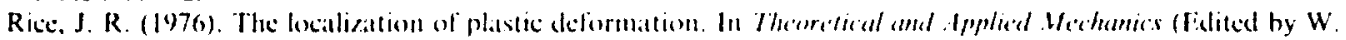
T. Koiter). pp. 155 lot. North-llolland, Amsterdim.

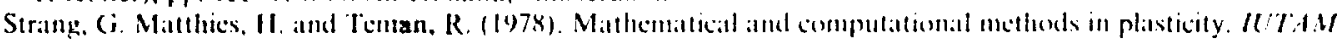

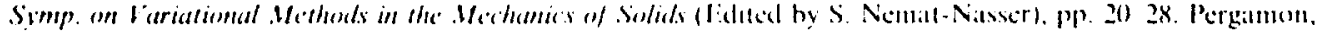
Oxford.

Temam, R. (1984). Mathematical Prohblems in I'lasticits. (iatuther Villats, Matris.

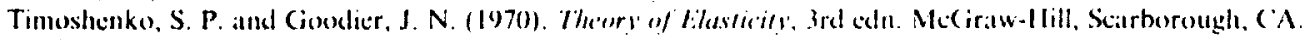

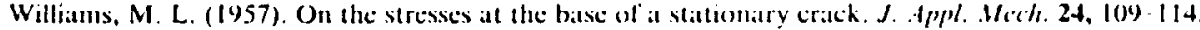

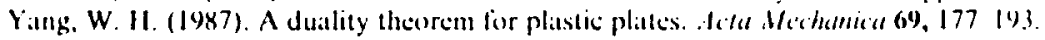

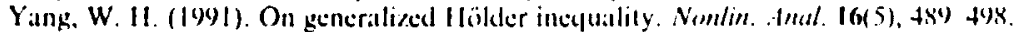

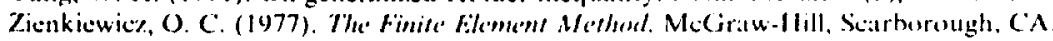

